A Small Country in Big Science

\section{Helsinki Institute of Physics: Strengthening National and International Collaboration}

\author{
by its director Eero Byckling
}

The membership fee of Finland is a small fraction of the total CERN budget and its financial participation in the LHC experiments will be on roughly the same relative level. How can a small country obtain significant visibility in large international projects to justify its participation? In Finland a new organisational structure has been created as one response to this challenge. The aim is to increase collaboration between universities and between physics and technology. As a consequence, the content of our CERN-related research is gradually changing.

The Helsinki Institute of Physics (HIP) was created in September 1996 by merging three institutes. These were the Research Institute for High Energy Physics (SEFT) and the Research Institute of Theoretical Physics (TFT) from the University of Helsinki, and the Institute of Particle Physics Technology (HTI) from the Helsinki University of Technology. The new organisation was given the responsibility for Finland's collaboration with CERN and other international accelerator centres. Its charter is to carry out research on particle physics, on any fields of theoretical physics, and on accelerator related technology. HIP also collaborates with universities by supporting graduate education.

A characteristic feature of Finnish physics, and also more generally of university education, is the fruitful interaction of basic research with technology and industry. This has been noted in studies of the national science policy and has often been commented on favourably in connection with the Finnish-CERN activities. To take one more step along this path, HIP has been structured as the first institute in Finland which is equally governed by two universities: the largest traditional multidiscipline university in Finland and the strongest technical university. Since Finland joined CERN in 1991, there has already been a close collaboration between these two universities in physics research. Another sign of the ties over traditional boundaries has been the company Cerntech. It has promoted the coupling of the Finnish industry to CERN and has

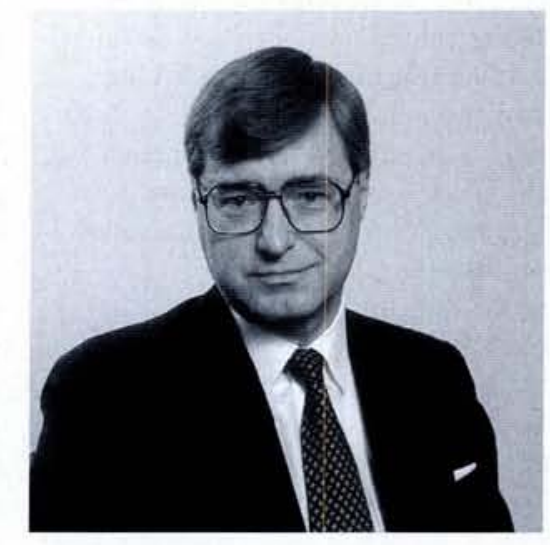

been active in transmitting calls for tender to industry and in initiating joint technology projects. During the last few years the Cerntech model has been copied to other CERN member countries: Hungary,

Netherlands, Poland, Portugal and Sweden.

\section{Flexibility and Focusing}

Collaboration is not sufficient. The level of science depends strongly on successful recruitment of talented people. Education and the research environment should be flexible enough so that these people are attracted to the most rewarding research topics. Experience shows that there are many obstacles in trying to achieve this. Typically, research groups are strongest and most influential several years after they have made important discoveries, not before them. The forefront research must often battle with outdated but still powerful research groups. As someone said: "The measure of man is not how many years he is ahead in his field, but how many years he retards its development."

Another factor contributing to the con- servatism of science is that universities have a tendency towards self-breeding. A scientist is working in a chosen field because in his or her mind it is the most interesting topic in all of science. Thus his or her students, and his or her successor when he or she retires, should, of course, represent the same field. High energy physics changes rapidly and active researchers should continuously be able to adjust. The organisational structures should support this adaptation.

For this reason, during the planning of the Helsinki Institute of Physics, considerable emphasis was placed on finding mechanisms for flexibility. Universities are responsible for the long-term stable support of various scientific fields. HIP has undertaken another task; it picks out the most topical fields of research in high energy physics, general theoretical physics and accelerator-related technology and focuses on these. There are, of course, many constraints in trying to accomplish this. In theoretical work the overwhelming requirement is to have talented people and to achieve a suitable mix of senior scientists with international reputation and bright young graduate students. In experimental work the access to suitable equipment and other facilities is crucial. Technological development work needs real interaction with industry.

Presently all appointments in HIP are for a limited period, typically 1-3 years. Some senior people are on a leave of absence from universities and some are only part-time in HIP. The freedom to concentrate on research in favourable circumstances is often sufficient to attract people with established careers to nonpermanent positions. However, it is clear that once the institute stabilises, long-term and permanent appointments are needed. This view was emphasised by the Scientific Advisory Board of HIP in its first meeting in September 1997. High level scientists cannot be attracted without the possibility of offering permanent posts.

\section{Avoiding Conflicts}

Often research institutes are steered by inviting physicists from various institutions and sub-fields of physics to a governing body which then decides on major matters concerning the institute. This type of organisation has the advantages of good coupling to surrounding universities, expertise in a number of physics research areas, and useful outside perspectives into the functioning of the institute.

Often, however, this structure brings 


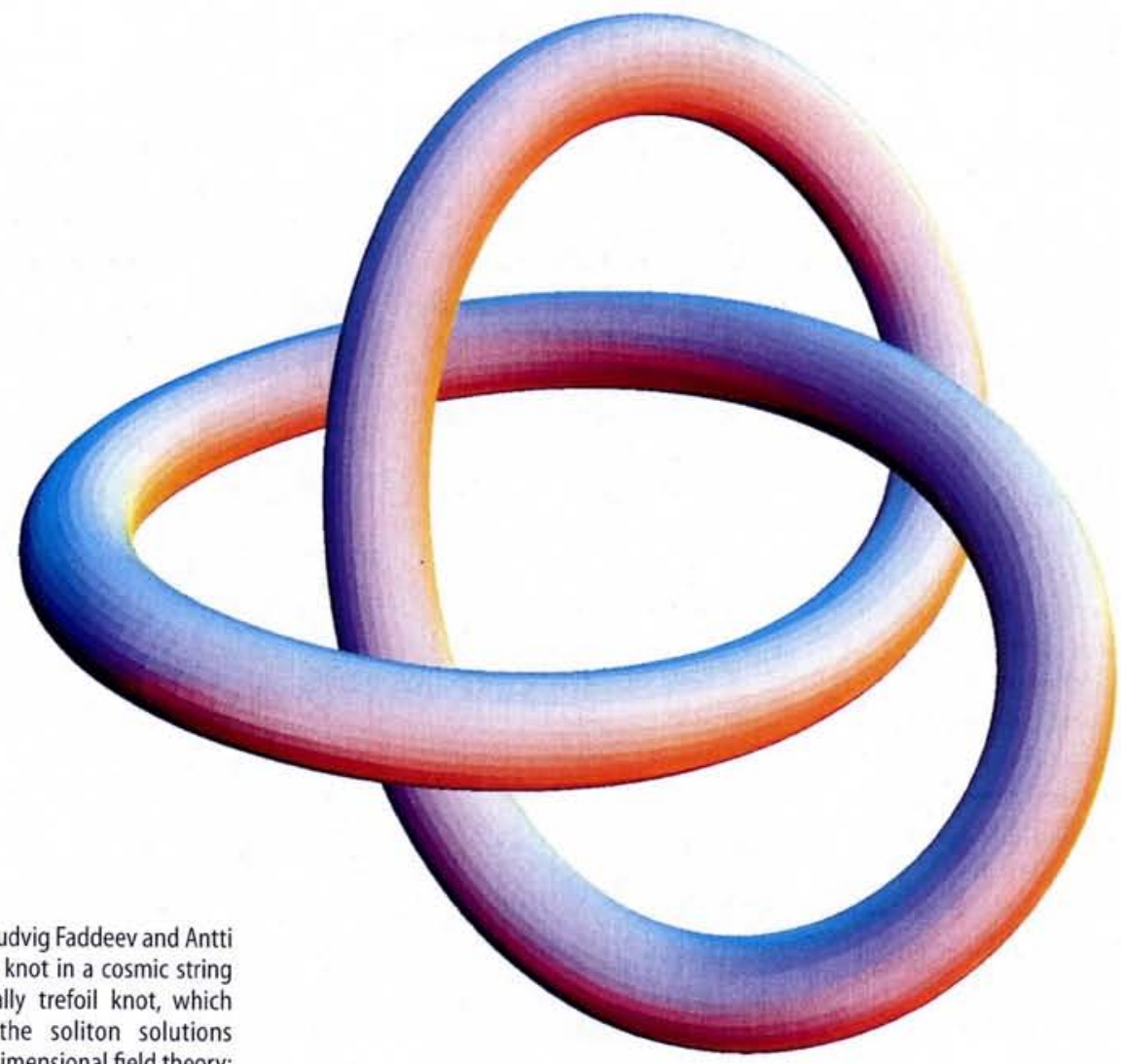

In May last year two physicists, Ludvig Faddeev and Antti Niemi, worked out how to tie a knot in a cosmic string (here shown as an energetically trefoil knot, which describes the structure of the soliton solution investigated in a realistic $3+1$ dimensional field theory; see Nature 387 p58)

also considerable problems. The members feel that they have been elected to safeguard the interests of their own background organisations and not primarily to help the institute to develop into a strong independent entity. In HIP the aim was to set up an administrative structure which would avoid conflicts with special interest groups while at the same time being able to use high level expertise in physics research.

The formula eventually chosen consists of a small (six member) Board which includes people with administrative experience. Presently the members are: the vice-rectors from both host universities, the physics department chairmen from the two universities, a member appointed by the Ministry of Education, and a representative of the personnel. In addition, the rectors of the two universities have appointed a six-member Scientific Advisory Board, which consists of five internationally recognised foreign scientists with in-depth knowledge of physics, and also an influential representative from the Finnish industry. The Finnish member is chosen to bring forward views on the technology projects at HIP. This administrative structure has already attracted favourable comments and it has been sug- gested that one should change some other Finnish institutes to adopt a similar mode of operation.

\section{Research at HIP}

During the first year of operation, the research at HIP has been directed towards the same topics as were active in the three previous institutes. In September 1997 the Scientific Advisory Board drafted in their meeting seven recommendations which were along similar lines that had already been discussed inside HIP. Thus, a firm basis was provided for clear priorities in research. In addition, the $\mathrm{SAB}$ also emphasised the need for long-term and permanent positions, need for appropriate laboratory facilities, and desirability of a chair in experimental particle physics at the University of Helsinki.

The research at HIP will increasingly be concentrated on three topics.

- The responsibility for the collaboration with CERN means that the largest single part of resources will be aimed at guaranteeing an eventual participation in the LHC experiments. Finnish scientists were among the originators of the CMS experiment and our contributions will mainly involve software, calibration, mechanics, and data acquisition. HIP will also take part in the Alice experiment and to a small extent in Atlas.

- Theoretical research has traditionally been the most visible part of Finnish physics and active groups are envisaged in field theory, non-linear physics, laser physics, quantum optics, and statistical physics.

- The technology group has developed the well-known Tuovi project and document management software for handling the construction of the LHC accelerator and the three experiments. This system is also being implemented at DESY and the MaxPlanck Institute and developed further.

In this brief exposition the most important matter, research results obtained at HIP, cannot be given its due. I shall only indicate one interesting result among many which could be cited. Academician Ludwig Faddeev and Antti Niemi have recently numerically constructed knot solutions to the Faddeev model and this has received wide attention as the first solution of the problem of knots in field theory (see above). This theory has a wealth of possible applications, both in particle physics and in low energy physics. The above picture symbolises the first major results emerging from the Helsinki Institute of Physics. 